\title{
Seizures Induce Dramatic and Distinctly Different Changes in Enkephalin, Dynorphin, and CCK Immunoreactivities in Mouse Hippocampal Mossy Fibers
}

\author{
Christine Gall \\ Department of Anatomy and Neurobiology, University of California, Invine, California 92717
}

\begin{abstract}
Light microscopic immunocytochemical techniques were used to evaluate the influence of recurrent limbic seizure activity on the immunoreactivity for 3 neuropeptides-enkephalin, dynorphin, and cholecystokinin (CCK)-contained within the mouse hippocampal mossy fiber axonal system. Seizures were induced either by the placement of a small unilateral electrolytic lesion in the dentate gyrus hilus or by intraventricular injection of kainic acid. Both treatments induce epileptiform activity in hippocampus lasting several hours. Four days after either lesion placement or injection of 0.05-0.1 $\mu \mathrm{g}$ kainic acid, immunoreactivity for all 3 peptides was altered throughout the intact mossy fiber system, bilaterally, but in distinctly different ways: enkephalin immunoreactivity (ENK-I) was dramatically elevated, dynorphin immunoreactivity was reduced, and CCK immunoreactivity (CCK-I) was either severely reduced or completely absent in the mossy fiber system. ENK-I was also clearly increased in other areas, including the lateral septum, the entorhinal cortex, and within the entorhinal (perforant path) efferents to temporal hippocampus. In contrast, the loss of CCK seemed restricted to the mossy fiber system in that immunostaining appeared normal in scattered hippocampal perikarya, within the dentate gyrus commissural system, as well as within other limbic structures. Four days after injections of 0.2 or $0.25 \mu \mathrm{g}$ kainic acid, mossy fiber ENK-I was greatly elevated, dynorphin immunoreactivity was reduced, but, unlike the situation with lower kainic acid doses, CCK-I was only modestly reduced in the mossy fibers and was clearly reduced in other hippocampal systems as well. These data indicate that epileptiform physiological activity differentially affects the regulation of 3 neuroactive peptides contained within the hippocampal mossy fiber system and suggest a mechanism through which seizurelike episodes can have a lasting influence on the operation of specific hippocampal circuitries.
\end{abstract}

There is now a significant body of evidence indicating that the synthesis of neuroactive substances by neurons and other secretory cells can be modified by a variety of experimental manipulations, including disturbances in the normal patterns of

\footnotetext{
Received Mar. 17, 1987; revised Aug. 14, 1987; accepted Oct. 20, 1987.

I would like to thank Dr. E. Weber for supplying antisera to dynorphin B, Dr. L. Terenius for supplying antisera to dynorphin A (1-17), Dr. G. Lynch for his comments on the manuscript, and J. C. Lauterborn for technical assistance. This work was supported by NSF Grant BNS 8417098 and Research Career Development Award NS00915 to C.G.

Correspondence should be addressed to Dr. Gall at the above address.

Copyright $(C 1988$ Society for Neuroscience $0270-6474 / 88 / 061852-11 \$ 02.00 / 0$
}

physiological activity. Destruction of the olfactory nerve, for example, produces a marked reduction of the synthesis of dopamine and a depletion of its synthetic enzyme tyrosine hydroxylase in olfactory bulb neurons, an effect that reverses upon regeneration of the nerve (Nadi et al., 1981; Kawano and Margolis, 1982; Baker et al., 1983). Elimination of normal sensory input to the visual cortex by enucleation or eyelid suture causes a transynaptic reduction of the GABA biosynthetic enzyme glutamic acid dccarboxylase (GAD) in the deprived-eye ocular dominance columns of monkey striate cortex (Hendry and Jones, 1986). More direct evidence for the role of synaptic activity in the differential regulation of the synthesis of transmitters and neuropeptides has come from studies on the adrenal medulla. Adrenal medullary cells are known to store and release both catecholamines and leucine enkephalin (Wilson et al., 1982). In rat, electrical stimulation of splanchnic nerve accelerates tyrosine hydroxylase activity and, consequently, increases catecholamine production by the adrenal cells (Unger and Phillips, 1983). Conversely, decreased synaptic activation by splanchnic denervation, pharmacological blockade, or explantation to culture results in increased adrenal leucine enkephalin content (Kilpatrick et al., 1984; LaGamma et al., 1984). LaGamma and colleagues (1986) further demonstrated that the increase in enkephalin synthesis (as measured by level of preproenkephalin mRNA or increases in leucine enkephalin content) obscrved with explantation of rat adrenal to culture is blocked by the presence of depolarizing concentrations of potassium or veratridine. In contrast, Kanamatsu et al. (1986c) recently reported that reflex activation of the splanchnic nerve by insulin-induced hypoglycemia increases enkephalin synthesis and content in rat adrenal medulla.

Much of the evidence that peptide levels in brain are influenced by changes in physiological activity comes from studies showing that seizures affect peptide content and synthesis (Sperk et al., 1986). The opioid peptides have been most extensively studied in this regard. Several treatments that induce recurrent or prolonged seizure activity, including repeated electroconvulsive shock (ECS) (Yoshikawa et al., 1985; Kanamatsu et al., 1986a), intracerebral kainic acid (Hong et al., 1980; Gall et al., 1981 b; McGinty et al., 1983; Kanamatsu et al., 1986b), and chemical and electrical kindling (Vindrola et al., 1981, 1984; Iadarola et al., 1986; McGinty et al., 1986) have been found to elevate enkephalin levels in a number of brain areas, with some differences in the regional distribution of increased enkephalinlike immunoreactivity (ENK-I) between treatments. Work in this laboratory has demonstrated that recurrent seizure activity induced by small electrolytic lesions in the hilus of the dentate 


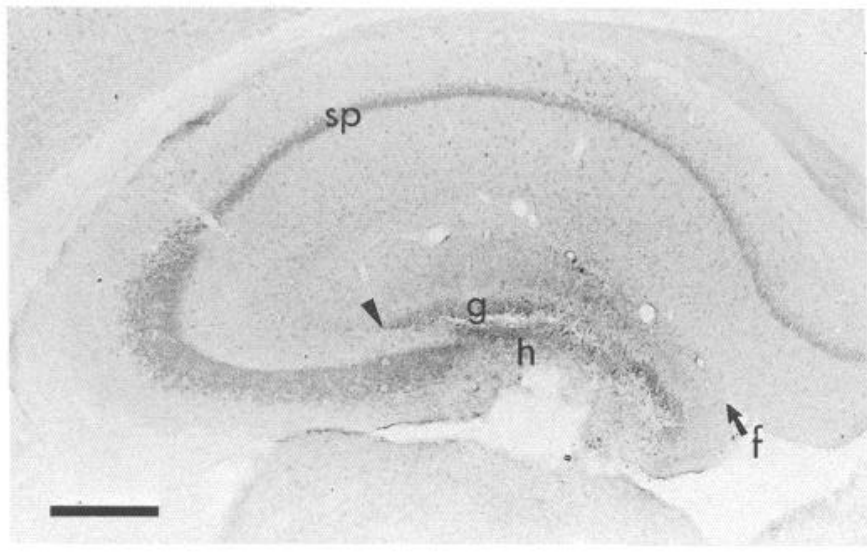

Figure 1. Low-magnification photomicrograph of a coronal section through the rostral hippocampus, processed for localization of dynorphin immunoreactivity, illustrating the extent and placement of the hilus lesion used in the present study. Peroxidase-antiperoxidase (PAP) technique. Abbreviations: $f$, hippocampal fissure; $h$, dentate gyrus hilus; $s g$, stratum granulosum; $s p$, stratum pyramidale. Arrowhead indicates the limit of the internal, suprapyramidal, blade of the granule cell layer. Scale bar, $300 \mu \mathrm{m}$.

gyrus (Gall et al., 1988) leads to large increases in hippocampal ENK-I, most prominently within the hippocampal mossy fiber system (Gall et al., 1981a, b; White et al., 1987; see Gall, 1988, for review). In contrast, the influence of seizures on dynorphin immunoreactivity has been less consistent; instances of increase (Iadarola et al., 1986; Kanamatsu et al., 1986b) and decrease (Iadarola et al., 1986; Kanamatsu et al., 1986a; McGinty et al., 1986) have both been reported.

The opioid peptides, and the enkephalins in particular, are colocalized with other neuropeptides as well as with classical neurotransmitters in a number of cell types and synaptic populations throughout the CNS (Charnay et al., 1982; Zahm et al., 1985; Gall et al., 1987). It would be of interest to determine if seizures have comparable effects on all peptides contained within a given system or, instead, differentially influence the regulation of peptide species and thereby alter the relative peptide or peptide/transmitter balance. In the present study, this issue was examined in the mossy fibers of the mouse hippocampus, which are well suited for this purpose. These axons arise from the dentate gyrus granule cells, are restricted to a discrete lamina within the ipsilateral hippocampal formation, and possess a unique, well-characterized, and thus quite recognizable, morphology (Blackstad and Kjaerheim, 1961; Blackstad et al., 1970). Moreover, the mossy fibers of mouse, like those of primate (Gall, 1988), contain enkephalin-, dynorphin-, and cholecystokinin octapeptide (CCK)-like immunoreactivities (Gall et al., 1986). The results indicate that seizures differentially influence the cellular mechanisms regulating the levels of the 3 neuropeptides within this one axonal system. Since these peptides have epileptogenic properties, the observed perturbations suggest a mechanism by which seizures produce lasting changes in the excitability of hippocampus. Preliminary results from this study have been published in abstract form (Gall, 1984c).

\section{Materials and Methods}

Adult male Swiss Webster mice, greater than $60 \mathrm{~d}$ of age, were used throughout this study. Under ketamine/xylazine anesthesia $(0.66 \mathrm{mg}$ ketamine, $0.13 \mathrm{mg}$ xylazine $/ 10 \mathrm{gm}$ body weight), experimental animals received either a small unilateral electrolytic lesion $(0.6 \mathrm{~mA}, 4 \mathrm{sec}$, via an insulated stainless steel wire) in the dentate gyrus hilus $(n=14)$ (Fig. 1) or an intracerebroventricular (i.c.v.) injection of $0.05-0.15 \mu \mathrm{g}(n=$ 8) or $0.20-0.25 \mu \mathrm{g}(n=3)$ kainic acid $(0.5 \mu \mathrm{g} / \mu \mathrm{l}$ in isotonic saline) placed in the lateral ventricle at the level of the foramen of Monro. Paired control mice $(n=14)$ were similarly anesthetized, but no surgery was performed. All mice were observed for the presence of seizure behavior for at least $4 \mathrm{hr}$ posttreatment. Within 1 and $2 \mathrm{hr}$, mice treated with either the hilus lesion or low doses of kainic acid exhibited intermittent forelimb and facial clonus, sudden head or body jerks, and nodding. These modest signs of seizure activity were followed by occasional stage 4 (rearing with facial and forelimb clonus) or stage 5 (full body and limb clonus and tonus followed by running and hopping) convulsive seizures (Gall et al., 1988). Animals treated with high doses of kainic acid remained fairly immobile, with occasional turning and nodding, and exhibited occasional full convulsive seizures with body and limb tonus and clonus, opisthotonos and rolling. All experimental animals exhibited at least one stage 4 or stage 5 convulsive seizure (2-5 such episodes were generally observed).

Four days posttreatment, experimental and paired control mice were anesthetized with an overdose of sodium pentobarbital and intracardially perfused with $0.9 \%$ saline followed by $4 \%$ paraformaldehyde in $0.1 \mathrm{~m}$ phosphate buffer, $\mathrm{pH} 7.2$ (PB). The brains were removed from the cranium, placed in the fixative perfusate for $1-2 \mathrm{hr}$ at $4^{\circ} \mathrm{C}$, and then stored in $20 \%$ sucrose in $\mathrm{PB}$ overnight at $4^{\circ} \mathrm{C}$. Coronal tissue sections were cut through the hippocampal formation at a thickness of $30 \mu \mathrm{m}$ using a freezing microtome and collected into PB. Within $24 \mathrm{hr}$ of being cut, sections from each brain were processed for the immunocytochemical localization of methionine enkephalin, sulfated CCK, and, in most cases, dynorphin B or dynorphin A (1-17) using the peroxidase-antiperoxidase technique, modified from Sternberger (1979), as described in detail elsewhere (Gall et al., 1981a). Five rabbit-raised polyclonal antisera were used (Table 1). The antiserum to methionine enkephalin was raised in rabbit inoculated with Ala-Ala-Ala methionine enkephalin conjugated with glutaraldehyde to keyhole limpet hemocyanin (Gall, $1984 \mathrm{~b}$ ). All antiserum incubations were conducted in PB including $0.3 \%$ Triton X-100. Primary and secondary antiserum incubations contained $1 \%$ normal goat serum as well. The secondary antiserum (goat antirabbit IgG; Cappel Labs) was used at a dilution of 1:50 and the peroxidase-antiperoxidase (DAKO) was used at a dilution of 1:200. Identical results were obtained with either antiserum to CCK.

Tissue sections from experimental and paired control animals were processed through the immunocytochemical procedure simultaneously to assure equivalent staining conditions for comparison purposes. This included the experimental and control sections being taken through the same diaminobenzidine solution in different compartments of a tissue basket.

The specificity of immunostaining obtained with each antiserum was tested by preadsorption with both homotypic and heterotypic antigens (see Table 1). For each of the 5 antisera used, immunoreactive staining was absent in tissue processed with antiserum that had been preadsorbed with $50 \mu \mathrm{M}$ of the homotypic antigen for $18-24 \mathrm{hr}$ at $4^{\circ} \mathrm{C}$. In all cases, preadsorption with $50 \mu \mathrm{M}$ of each of the heterotypic antigens failed to affect immunostaining. It is particularly important to note that methionine ENK-I in both control and experimental hippocampi was unaffected by preadsorption with $50 \mu \mathrm{M}$ dynorphin A (1-13), dynorphin A

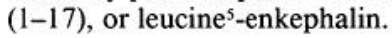

\section{Results}

\section{Normal immunostaining}

The distribution of dynorphin-like immunoreactivity and ENK-I in the untreated mouse hippocampus is essentially the same as has been reported for the rat (Gall et al., 1981a; McGinty et al., 1983). Dynorphin A- and dynorphin B-like immunoreactivities were localized exclusively within the mossy fiber system. As such, dynorphin immunoreactivity filled the hilus and stratum lucidum of regio inferior, the narrow dendritic layer containing the mossy fiber axons and terminals. With both antisera, immunostaining within stratum lucidum was localized within the fine axons and spherical swellings, presumably mossy fiber ter- 
Table 1. Antisera used

\begin{tabular}{lll} 
Antiserum to & Dilution & Supplier \\
\hline Cholecystokinin 8 & $1: 300$ & Immunonuclear Corp. \\
Cholecystokinin 8 & $1: 500$ & G. Rosenquist (Dept. Physiology, U.C. Davis) \\
Dynorphin A (1-17) & $1: 1000$ & L. Terenius (Uppsala, Sweden) \\
Dynorphin B & $1: 400$ & E. Weber (Oregon Health Sci. Univ.) \\
Methionine enkephalin & $1: 1000$ & Raised in collaboration with M. Cillufo \\
& & and N. Brecha (U.C.L.A.)
\end{tabular}

Preadsorption controls were conducted using all antigens listed above plus leucine ${ }^{\mathrm{s}}$-enkephalin, Ala-Ala-Ala-leucine enkephalin, Ala-Ala-Ala-methionine enkephalin, and dynorphin A (1-13).

minal boutons of varying sizes, that entirely filled the lamina. Interestingly, the dentate gyrus granule cells, which give rise to the mossy fiber axons, did not exhibit dynorphin immunoreactivity in these animals.

In agreement with what has been reported for the distribution of ENK-I in a number of other animals (Gall et al., 1981a; Stengaard-Pedersen et al., 1983), ENK-I in the mouse hippocampus was localized within sparsely scattered neuronal perikarya in all subfields, within a variable number of characteristic granule cells in stratum granulosum of the dentate gyrus, and within sparsely scattered large bouton-like swellings throughout the mossy fiber field (hilus and stratum lucidum). Unlike dynorphin immunostaining, mossy fiber ENK-I in the untreated mouse did not fill stratum lucidum (the ENK-I boutons were well separated by nonimmunoreactive space) and was rarely seen within the fine mossy fiber axons. Finally, a diffuse band of ENK-I was observed in the distal two-thirds of the temporal dentate gyrus molecular layer. This immunoreactivity is prcsumably localized within afferent axons from the lateral entorhinal cortex (the lateral perforant path) (Gall et al., 1981a; Fredens et al., 1984). Compared with findings in the rat, ENK-I staining of this system in the mouse was relatively faint and more restricted to the temporal dentate gyrus.

The distribution of CCK-I in the mouse hippocampus was found to be substantially different than that reported for other animals (Stengaard-Pedersen et al., 1983; Gall, 1984a), including rat (Greenwood et al., 1981), and has been described in detail in a separate report (Gall et al., 1986). Briefly, in untreated mice CCK-I is localized within sparsely scattered perikarya in all subfields of the hippocampus proper and immediately deep to the granule cell layer in the hilus of the dentate gyrus. In addition, CCK-I is localized within 3 principal axonal systems. First, immunoreactive axons and large terminallike varicosities are distributed around the pyramidal cell somata of stratum pyramidale in both regio inferior and regio superior. As in the rat, these presumably represent the axonal arborizations of the CCK-I local circuit neurons. Second, a dense band of fine granular CCK-I fills the proximal $28 \%$ of the dentate gyrus molecular layer along its entire septotemporal extent. This represents CCK-I localized within the commissural and ipsilateral associational projections of CCK-I neurons within the dentate gyrus hilus (Gall et al., 1986). Finally, CCK-I is found in the fine axons and terminal boutons of the mossy fiber projections throughout the hilus and stratum lucidum of hippocampal region $\mathrm{CA} 3$. As with dynorphin immunoreactivity, in the untreated mouse CCK-I fills stratum lucidum and appears to be present in boutons of varying sizes. It is noteworthy that CCK-I is not present in the mossy fiber system of the rat, while it is clearly evident in this system in the Swiss Webster mouse. In this regard, the intra- hippocampal distribution of CCK in mouse is similar to that obscrved in guinca pig, hedgehog (Stengaard-Pedersen et al., 1983; Gall, 1984a), and monkey (Gall, 1988) but differs from that of gerbil, rabbit, and cat (unpublished observations).

\section{Hilus lesion}

As illustrated in Figure 1, the hilus lesions used in the present study destroyed a narrow rostrocaudal span of the dentate gyrus hilus, portions of stratum granulosum, and stralum pyramidale of regions $\mathrm{CA} 3 \mathrm{c}$, and, sometimes, $\mathrm{CA} 3 \mathrm{~b}$. A span of the highly topographic and exclusively ipsilateral mossy fiber projection was severed by this lesion, and there was a complete loss of all peptide immunostaining within the narrow zone of mossy fiber degeneration. No pyknotic nuclei, indicative of neuronal degeneration, were observed in the hippocampus contralateral to lesion placement. Furthermore, electron microscopic studies in rat and silver impregnation of axonal degeneration in mouse (R. Pico and C. Gall, unpublished observations) have found no evidence for mossy fiber degeneration within hippocampus contralateral to the placement of such seizure-producing electrolytic hilus lesions.

Following seizures induced by both the unilateral electrolytic lesion of the dentate gyrus hilus and intraventricular kainic acid (described below), dramatic changes in immunoreactivity for the 3 neuropeptides under examination were observed throughout all intact mossy fibers, bilaterally. However, to avoid confusion with degeneration/deafferentation effects ipsilateral to treatment, the following description of changes in immunoreactivity within experimental animals will focus on the contralateral hippocampus, where deafferentation is minimal (see Discussion) and the mossy fiber axonal system is entirely intact.

A large bilateral elevation of ENK-I staining of the mossy fibers was evident in all mice killed $4 \mathrm{~d}$ after hilus lesion placement (Fig. 2). In comparison with paired controls, there was an increase in both the number and staining intensity of ENK-I mossy fiber boutons in stratum lucidum and the dentate gyrus hilus (Fig. 2). The fine mossy fiber axons could be seen to contain immunoreactivity only in experimental animals. While not nearly so dramatic as the alteration in the immunoreactive staining of the mossy fibers, ENK-I in the perforant path afferents to the temporal dentate gyrus molecular layer was clearly increased, bilaterally, in lesioned mice relative to paired controls as well. Although not the focus of the present study, increased ENK-I was also noted in other brain areas, including the entorhinal cortex (Fig. 3), the amygdaloid complex, and the lateral septum.

In contrast to the increase in mossy fiber ENK-I, both dynorphin A- and dynorphin B-like immunoreactivity were reduced bilaterally in the intact mossy fibers of 7 of the 9 hiluslesion mice evaluated (Fig. 4). This reduction did not seem to 


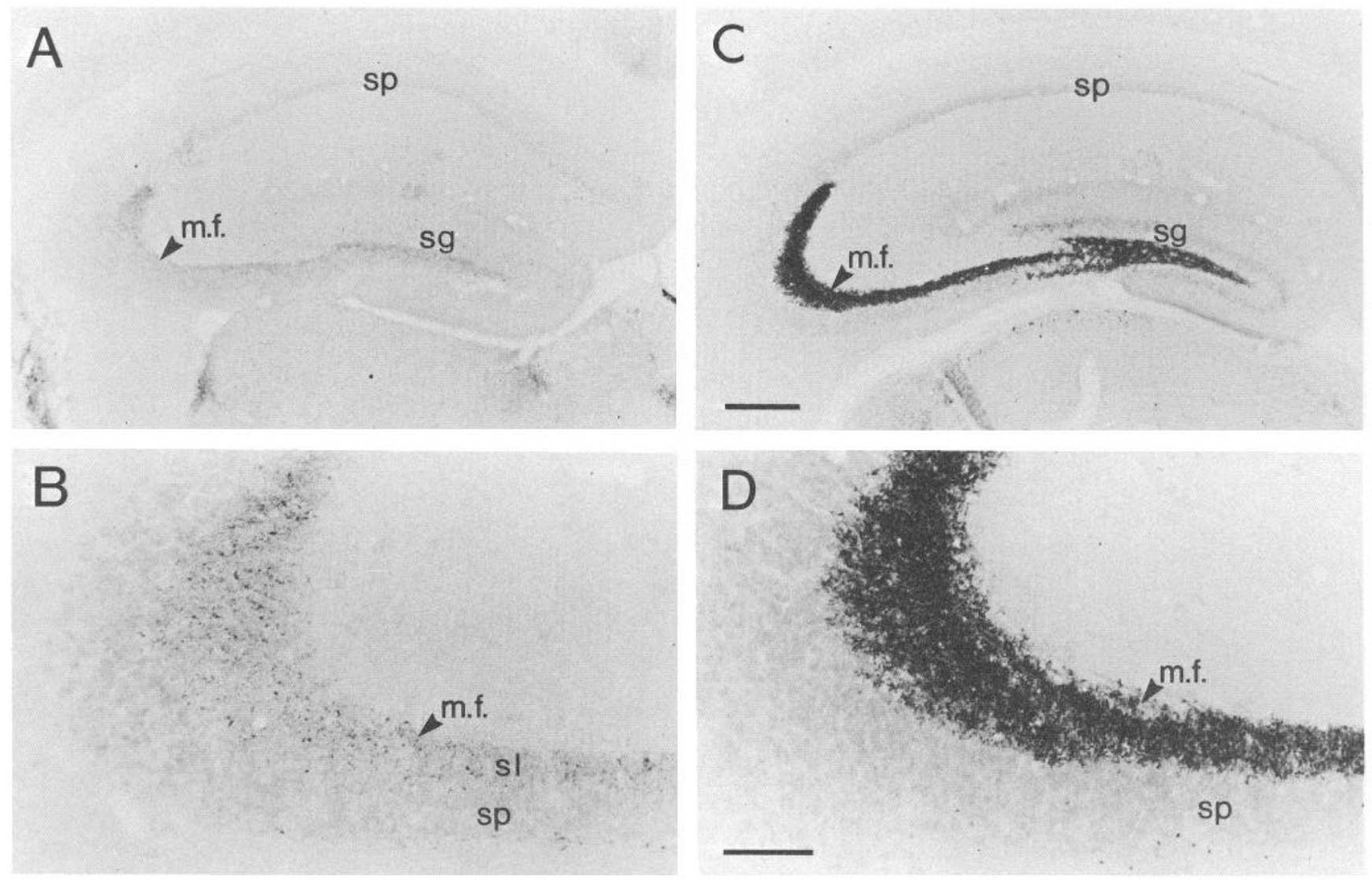

Figure 2. Photomicrographs illustrating the distribution and density of PAP-labeled ENK-I in hippocampus of a normal mouse $(A, B)$ and a paired experimental mouse sacrificed $4 \mathrm{~d}$ after a contralateral hilus lesion $(C, D) . A$ and $C$, Low-magnification photomicrographs show a full coronal cross section through the rostral hippocampus. $B$ and $D$, Region CA3 at higher magnification. Note the dramatic increase in ENK-I and the apparent increase in the number of ENK-I puncta within the mossy fiber system $(m . f)$ ) of the experimental animal. Abbreviations: $s g$, stratum granulosum; $s l$, stratum lucidum; sp, stratum pyramidale. Scale bars: $300 \mu \mathrm{m}$ in $C$ for $A$ and $C ; 100 \mu \mathrm{m}$ in $D$ for $B$ and $D$.

involve a change in the number of immunoreactive elements within stratum lucidum since dynorphin immunoreactive staining filled the zone in both experimental and control animals. Rather, there was a uniform but partial reduction in the intensity of staining. In these same animals, dynorphin immunoreactivity in some other brain areas, such as the substantia nigra, seemed unaffected by the hilus lesion.

CCK-I was virtually eliminated bilaterally in the mossy fiber system of all 12 mice with well-placed hilus lesions (Figs. 5, 6). This loss of immunoreactivity appeared to be specific to the mossy fibers. As described above, in addition to the mossy fibers, CCK-I in the mouse hippocampus is localized within the commissural/associational afferents to the dentate gyrus inner molecular layer, as well as within perikarya and pericellular axonal varicosities in the hippocampus proper. In the same hilus-lesion animals with complete depletion of mossy fiber CCK-I, immunoreactivity in these other areas appeared normal (Fig. 5).

The magnitude of these lesion-induced alterations in mossy fiber peptide immunoreactivity varied with the accuracy of lesion placement, and there was a good correlation between the magnitude of the change in immunostaining for each of the 3 peptides within contralateral hippocampus of a given animal. That is, mice with lesion placement within the hilus/CA3c region (as in Fig. 1) exhibited the most dramatic increase in mossy fiber ENK-I, complete depletion of mossy fiber CCK-I, and a clear reduction in dynorphin immunostaining. In the 2 cases in which the lesion was either particularly large (exceeding the limits of the dentate gyrus into regions $\mathrm{CA} 1$ and $\mathrm{CA} 3$ ) or lateral (placed directly over region CA3), the increase in ENK-I was submaximal, CCK-I was reduced but still present, and mossy fiber dynorphin immunoreactivity was not clearly affected.

\section{Kainic acid}

The response of hippocampal peptide immunoreactivity to treatment with kainic acid was somewhat more complex than the response to the hilus lesion. In particular, different effects were obtained depending on the amount of kainic acid injected. Four days after the injection of low doses of kainic acid $(0.05$ $0.15 \mu \mathrm{g})$, mossy fiber peptide immunoreactivity was altered in the same manner as observed following unilateral hilus lesions. As can be seen in Figure 6, there was a dramatic increase in ENK-I and a strong reduction in CCK-I in the mossy fiber system of these mice relative to simultaneously processed controls. This effect was bilateral and, as seen in hilus-lesion mice, the CCK-I staining of the dentate gyrus inner molecular layer and scattered neuronal perikarya was not detectably affected at these doses. Both dynorphin A- and dynorphin B-like immunoreactivities were reduced bilaterally in the mossy fibers following low-dose kainic acid as well, but this reduction was never so dramatic as that seen following an optimally placed hilus lesion. 

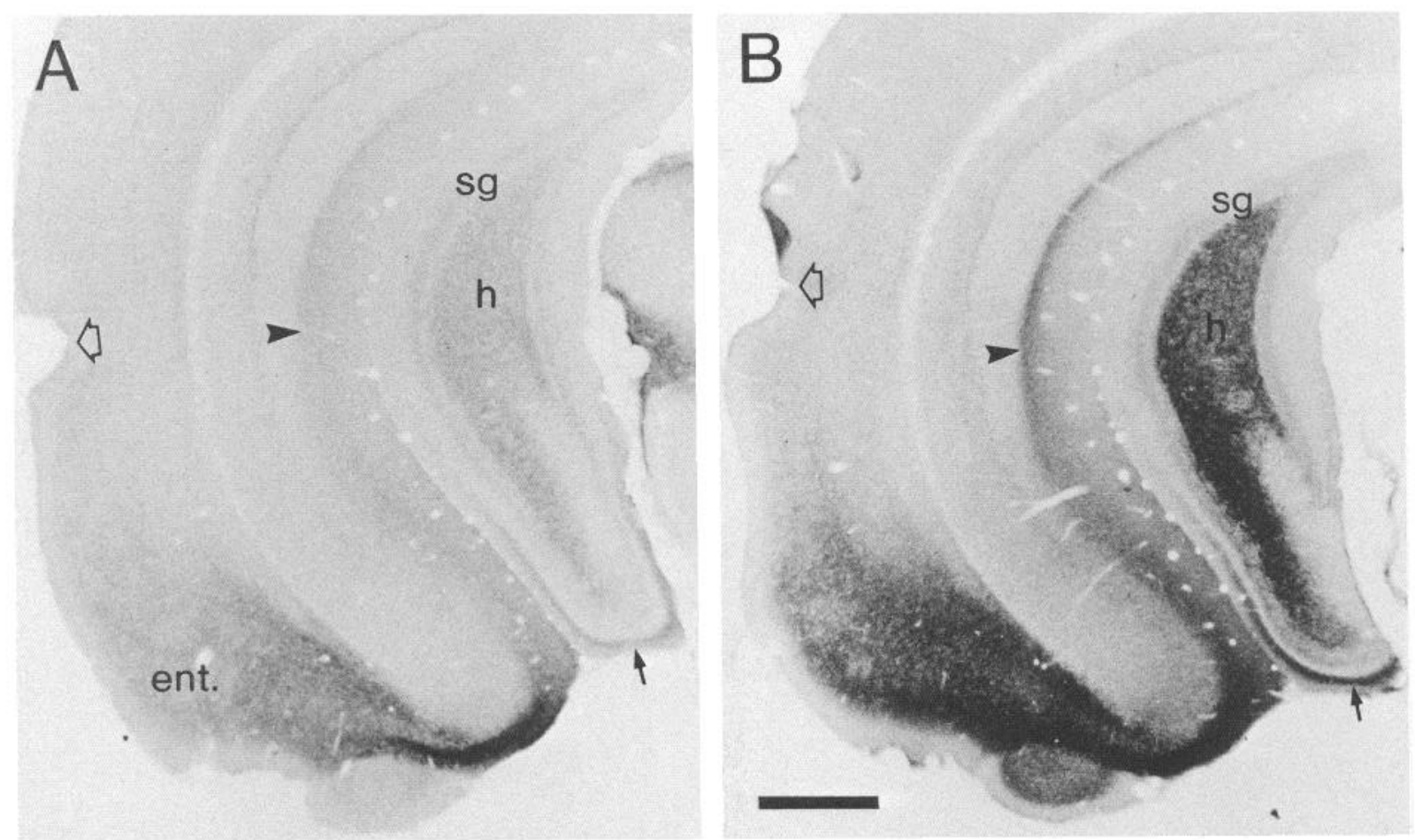

Figure 3. Photomicrographs illustrating the distribution and density of ENK-I in coronal sections through the entorhinal cortex (ent.) and adjacent temporal hippocampal formation of a control $(A)$ and a paired hilus-lesion mouse (side contralateral to the lesion shown; $B$ ). In $B$, note the relative increase in ENK-I within the entorhinal cortex as well as within the perforant path (small arrow) and temperoammonic (arrowhead) efferents of the entorhinal cortex which distribute within the dentate gyrus molecular layer and hippocampus proper, respectively. Elevated ENK-I within the mossy fiber system in the dentate gyrus hilus $(h)$ of the lesioned mouse is clearly evident as well. Open arrow indicates the rhinal fissure. $s g$, stratum granulosum. Scale bar, $400 \mu \mathrm{m}$.

In the 3 animals treated with high doses of kainic acid $(0.2$ and $0.25 \mu \mathrm{g}$ ), mossy fiber ENK-I was tremendously increased bilaterally, as with lower doses, while there was an overall but modest reduction of CCK-I staining, relative to paired controls, throughout both hippocampi. As such, CCK-I was reduced in the dentate gyrus inner molecular layer and was reduced, but not completely depleted, from the mossy fiber zone. Dynorphin immunoreactivity was not evaluated in these animals.

\section{Discussion}

The present results demonstrate that 2 limbic-seizure-producing treatments cause dramatic alterations in the amount of immunoreactivity for the 3 neuropeptides contained within the mouse hippocampal mossy fiber system. Four days after either an electrolytic lesion of the dentate gyrus hilus or i.c.v. injection of convulsant doses of kainic acid, there is a dramatic increase in ENK-I, a partial reduction of dynorphin immunoreactivity, and a complete or nearly complete elimination of CCK-I in this one axonal system. Similar alterations in mossy fiber ENK-I and dynorphin immunoreactivity have been observed following the application of these treatments in the rat (Gall et al., 1981b; Gall, 1988).

Several lines of evidence indicate that it is limbic seizure activity, rather than some other aspect of treatment, that is responsible for the alterations in peptide immunoreactivity in these cases. First, both electrolytic hilus lesion (Baudry et al., 1986; Gall et al., 1987b; R. Pico and C. Gall, unpublished observations) and i.c.v. kainic acid (Sloviter and Damiano, 1981) induce recurrent motor and bilateral hippocampal seizure activity lasting for several hours in rats and mice; and, while both treatments elicit a contralateral increase in enkephalin peptide within the mossy fibers and preproenkephalin A mRNA within the dentate gyrus granule cells when applied to rats under ketamine/xylazine or ether anesthesia, neither treatment is effective when applied to animals under deep, prolonged anesthesia with the anticonvulsant sodium pentobarbital (Hong et al., 1980; Gall et al., 1981b; White and Gall, 1987). Furthermore, the broad distribution of the observed changes in mossy fiber peptide immunoreactivity points to diffuse seizure activity as the triggering agent and certainly cannot be accounted for by the spatially restricted degeneration induced by each treatment. The lower doses of kainic acid used here eliminate a portion of the CA3 neurons in the ipsilateral rostral hippocampus. The unilateral hilus lesion directly destroys neurons in a narrow rostrocaudal span of the dentate gyrus and, to a variable extent, hippocampal regions $\mathrm{CA} 3 \mathrm{c}$ and $\mathrm{CA} 3 \mathrm{~b}$. Consequently, in the hippocampus contralateral to treatment one would expect to find axonal degeneration in the apical and basal dendritic fields of the rostral hippocampus proper (CA3-CA1) following kainic acid and in the proximal molecular layer of the rostral dentate gyrus following the hilus lesion (Fricke and Cowan, 1978; Laurberg, 1979). Neither treatment causes neuronal degeneration or deafferentation in the caudal half of the hippocampal formation of either side. Nor does either treatment cause degeneration of mossy fibers in contralateral hippocampus. In contrast, the alterations in immunoreactivity for the 3 peptides were observed in all intact mossy fibers, throughout the septotemporal extent of hippocampus, bilaterally.

The increase in mossy fiber ENK-I observed following the hilus lesion is consistent with other reports of delayed increases 


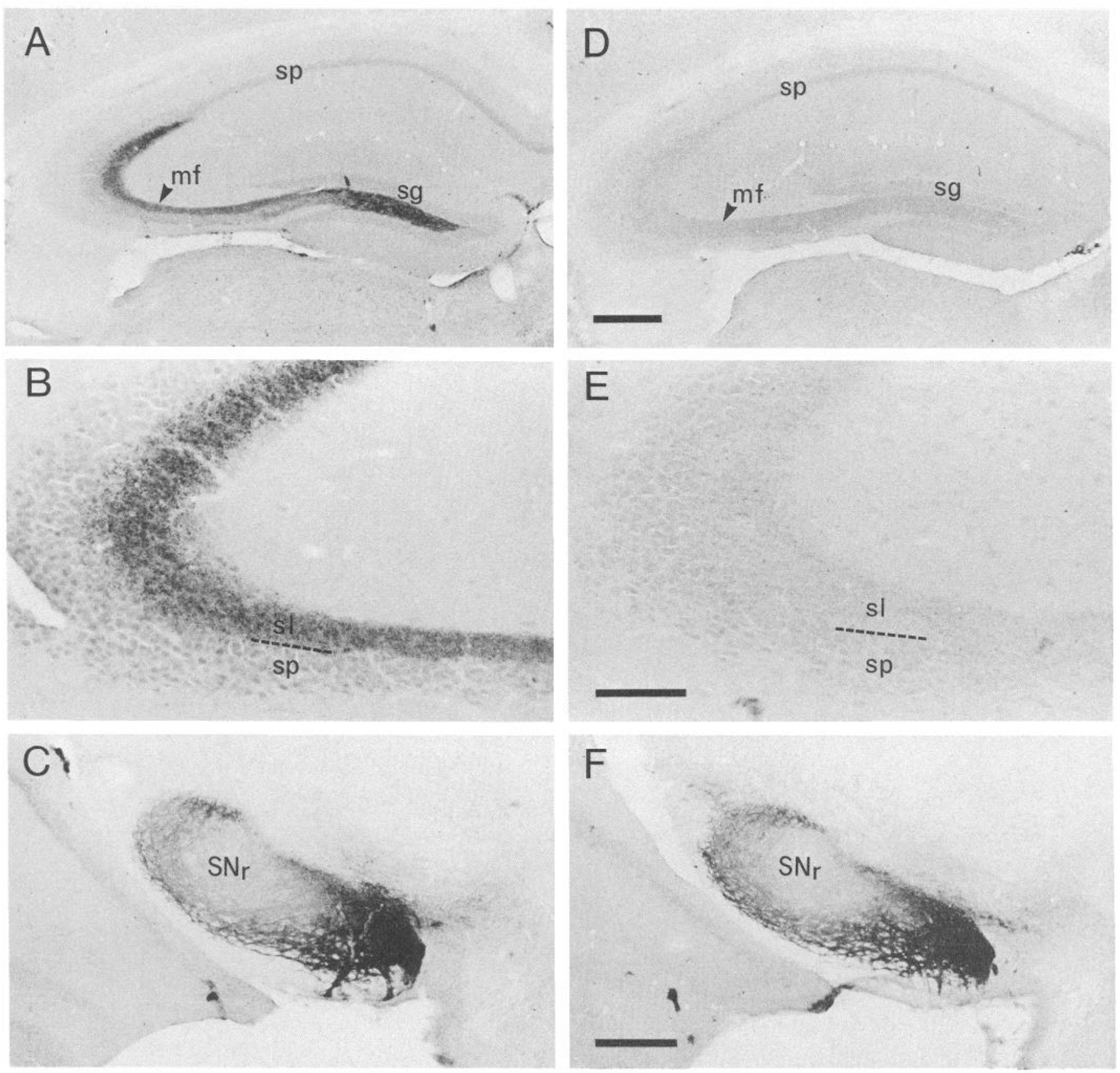

Figure 4. Photomicrographs of dynorphin B-like immunoreactivity in the hippocampus and substantia nigra of a normal mouse (A-C) and a mouse sacrificed $4 \mathrm{~d}$ after the placement of a contralateral hilus lesion $(D-F)$ that induced particularly severe seizure behavior. As can be seen in the low-magnification micrographs of $A$ and $D$, and at higher magnification in micrographs of region CA 3 in $B$ and $E$, dynorphin immunoreactivity within the mossy fiber system $(m f)$ is dramatically reduced, but not totally depleted, in the treated mouse relative to its paired control. There is no obvious alteration in dynorphin immunoreactivity in the substantia nigra of the same hilus-lesion mouse $(F)$ compared with the control $(C)$. Abbreviations: $S N r$, substantia nigra pars reticulata; $s l$, stratum lucidum; $s p$, stratum pyramidale. Scale bars: $300 \mu \mathrm{m}$ in $D$ for $A$ and $D$; $100 \mu \mathrm{m}$ in $E$ for $B$ and $E$; and $300 \mu \mathrm{m}$ in $F$ for $C$ and $F$.

in enkephalin content following recurrent seizures (Hong et al., 1980; Vindrola et al., 1981, 1984; Iadarola et al., 1986; Kanamatsu et al., 1986a, b; McGinty et al., 1986). Considered in this context, the effect of hilus lesion-induced seizures on hippocampal enkephalin is most like that of kainic acid (Gall et al., 1981b; Kanamatsu et al., 1986b; present data) in that both treatments most dramatically increase ENK-I in the mossy fiber system. In contrast, the somewhat more modest increases in hippocampal ENK-I seen after ECS or amygdaloid kindling are reported to be most pronounced within the temperoammonic and lateral perforant paths (Kanamatsu et al., 1986a; McGinty et al., 1986). It seems likely that seizures reliably increase ENKI, with the sites of the greatest effects depending upon the topography of epileptiform activity.

In contrast, epileptiform activity has been found to decrease or increase dynorphin immunoreactivity depending on brain region sampled, the posttreatment interval examined, and the mode of seizure induction. The reduction in mossy fiber dy- 

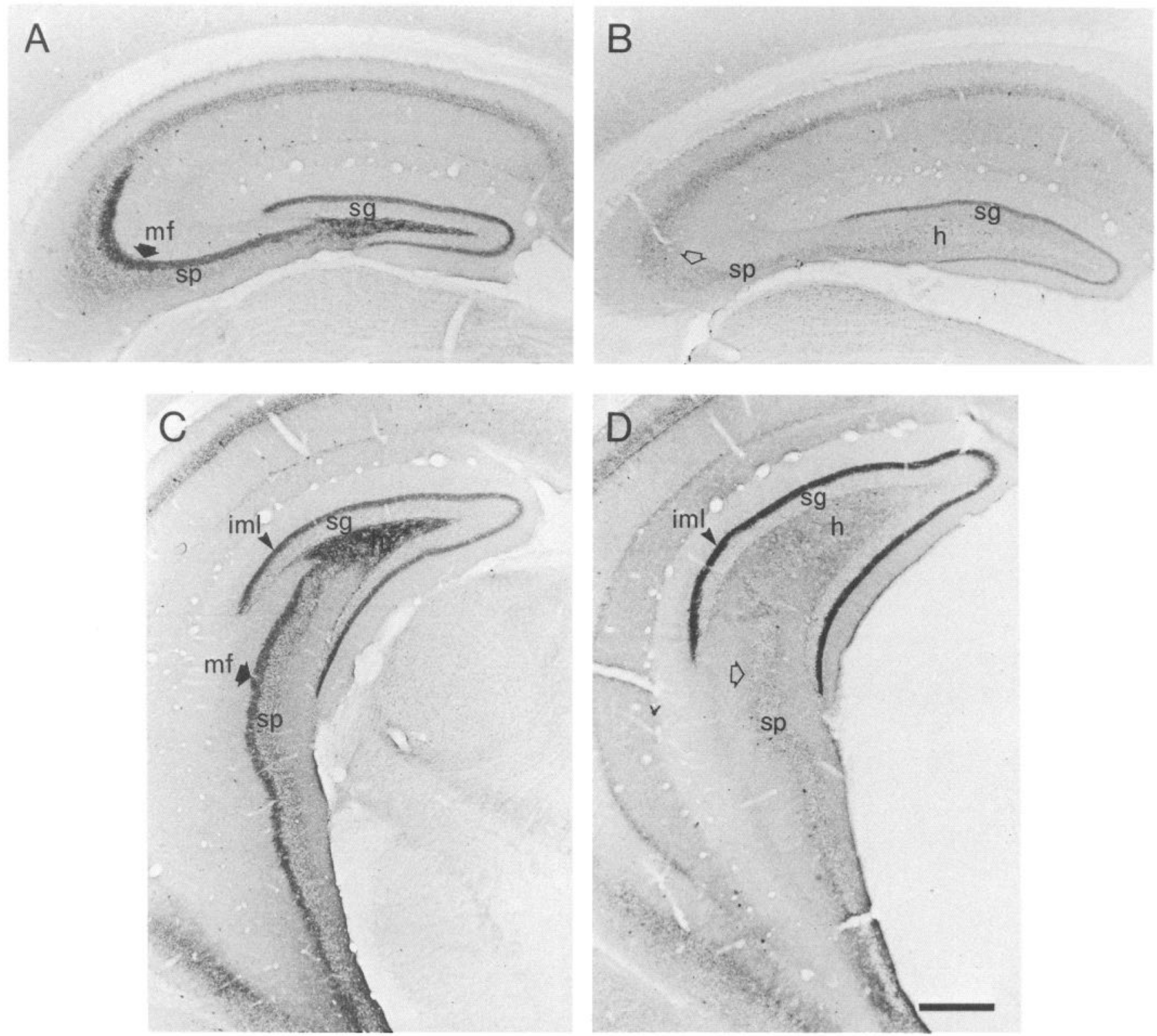

Figure 5. Low-magnification photomicrographs illustrating the distribution and density of PAP-labeled CCK-I in relatively rostral $(A, B)$ and caudal $(C, D)$ coronal sections through the hippocampus of an untreated mouse $(A, C)$ and a paired experimental mouse sacrificed $4 \mathrm{~d}$ after a contralateral hilus lesion $(B, D)$. Note the loss of CCK-I from the mossy fiber system $(m f)$ of the experimental animal (location indicated by open arrow $)$ and the retention of CCK-I within the dentate gyrus inner molecular layer $(\mathrm{im} l)$. Abbreviations: $h$, dentate gyrus hilus; $s g$, stratum granulosum; $s p$, stratum pyramidale. Scale bar, $400 \mu \mathrm{m}$.

norphin immunoreactivity seen here is in agreement with both our own observations on the effect of these treatments in rat (Gall, 1988) and previous reports of reduced hippocampal dynorphin following repeated ECS or amygdaloid kindling in rat (Kanamatsu et al., 1986a; McGinty et al., 1986). In contrast, high doses of kainic acid injected into rat striatum reportedly increase dynorphin in the mossy fibers at $2 \mathrm{~d}$, but not $4 \mathrm{~d}$, postinjection (Kanamatsu et al., 1986b); and kainic acid injected into the amygdala reportedly elevates total hippocampal dynorphin by $1 \mathrm{~d}$ postinjection (Lason et al., 1983). In further contrast to enkephalin, dynorphin levels in different brain areas respond differently to seizure activity. While dynorphin immunoreactivity is reduced in rat hippocampus following sei- zures induced by hilus lesion, i.c.v. kainic acid, kindling (Iadarola et al., 1986; McGinty et al., 1986), and repeated ECS (Kanamatsu et al., 1986a), it is reportedly increased in substantia nigra following kindling (McGinty et al., 1986) and in several brain areas (hypothalamus, substantia nigra, ventral pallidum, septum) following ECS (Kanamatsu et al., 1986a).

The present finding of prolonged depletion of CCK from the mouse mossy fibers following the hilus lesion or i.c.v. kainic acid represents, to our knowledge, the first report of a sustained reduction of CCK-I following seizure. Meyer et al. (1986) found that CCK-I is increased in rat hippocampus and substantia nigra following i.c.v. kainic acid, and we observed no change in preprocholecystokinin message in rat entorhinal cortex following 

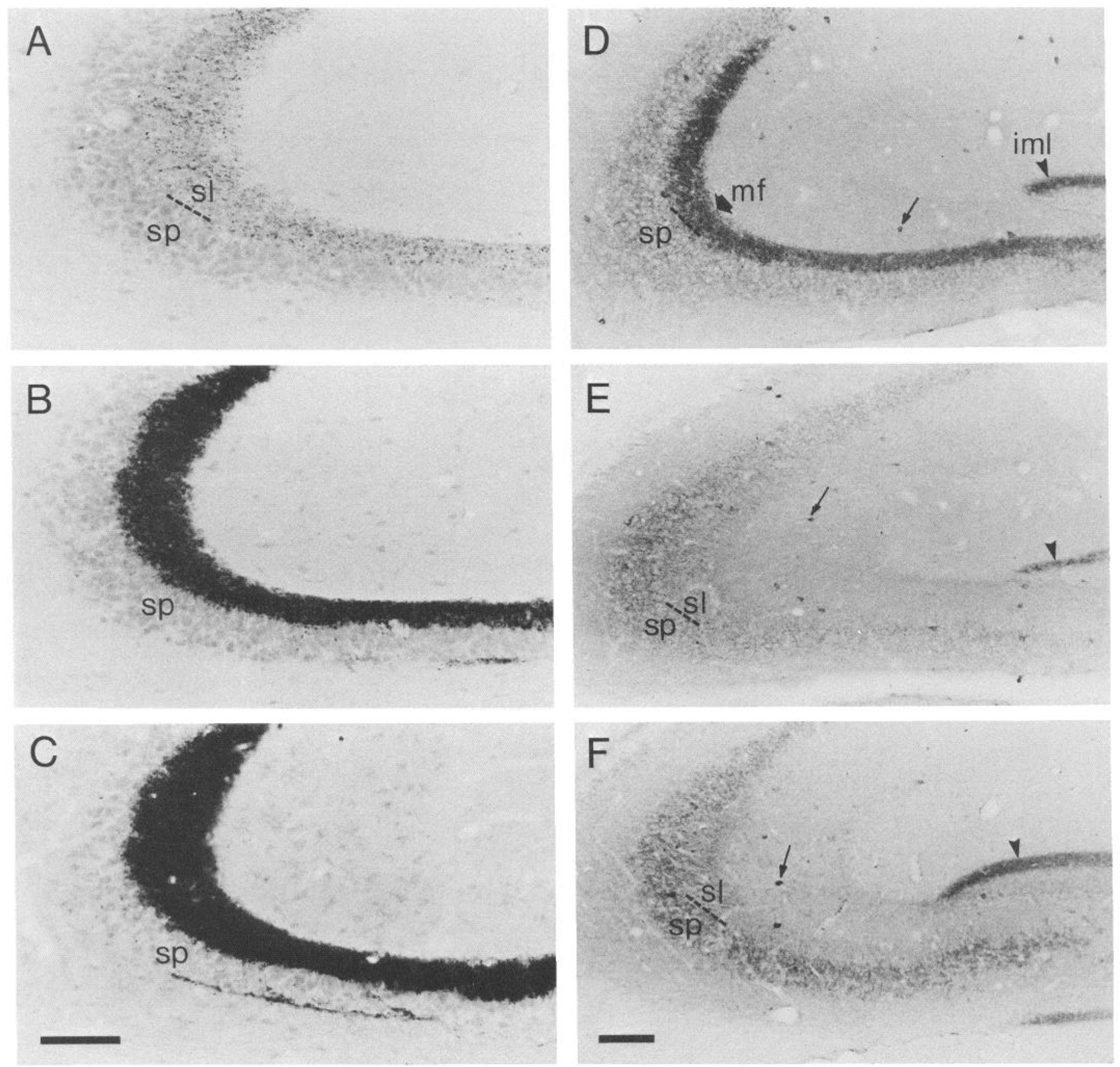

Figure 6. Photomicrographs illustrating the distribution and density of PAP immunostaining for ENK-I $(A-C)$ and CCK-I $(D-F)$ in hippocampal region CA 3 of a normal mouse $(A, D)$ and mice sacrificed $4 \mathrm{~d}$ after either a contralateral hilus lesion $(B, E)$ or a contralateral i.c.v. injection of 0.1 $\mu \mathrm{g}$ kainic acid $(C, F)$. Note, in both treated mice there is a dramatic increase in ENK-I, including an apparent increase in the number of ENK-I puncta and a loss of CCK-I within the mossy fibers $(\mathrm{mf})$ of stratum lucidum $(\mathrm{s} l)$. CCK-I appears normal within scattered perikarya $(s m a l l$ arrows) and within the dentate gyrus inner molecular layer ( $\mathrm{iml}$, arrowheads) in the experimental animals. $s p$, stratum pyramidale. Scale bars: $200 \mu \mathrm{m}$ in $C$ for $A-C ; 100 \mu \mathrm{m}$ in $F$ for $D-F$.

hilus lesion-induced seizures (White and Gall, 1987). It should be remembered, however, that the loss of CCK-I obtained in the present experiments was restricted to the mossy fiber system and that CCK is not localized within this axonal system in the rat (Greenwood et al., 1981).

The above studies demonstrate that the regulation of neuropeptide content in CNS neurons is strongly influenced by the changes in physiological activity that accompany recurrent seizures. The most striking, and unprecedented, aspect of the present results is the observation that 3 unrelated neuropeptides, codistributed within one axonal system, respond very differently to the same physiological episode. Although we do not yet know whether all 3 peptides are colocalized within individual granule cells and their mossy fiber axons, it is clear that each is contained within at least some proportion of this system, and we can assume that the granule cells containing each are similarly involved in the seizure activity. Data on the time course of the fluctuations considered here support this point: immunoreactivities to enkephalin and CCK are comparably depleted from the mossy fibers $12 \mathrm{hr}$ after recurrent seizure initiation by con- 
tralateral hilus lesion in mouse (Gall, 1988). However, despite a similar initial response to the seizures, mossy fiber enkephalin levels are greatly increased and CCK-I remains undetectable 4 $\mathrm{d}$ after treatment.

Recent work in this laboratory, and elsewhere, has demonstrated that the postseizure increases in ENK-I are largely attributable to increased synthesis without significant change in posttranslational processing (White ct al., 1987). The clcvation of ENK-I observed in hypothalamus following repeated ECS (Yoshikawa et al., 1985) and in hippocampus following intrastriatal kainic acid (Kanamatsu et al., 1986b) is associated with increased preproenkephalin A mRNA in these areas. We have demonstrated that, in rat, hilus lesion-induced seizures stimulate a rapid bilateral increase in preproenkephalin A mRNA in the dentate gyrus which is apparent at $3 \mathrm{hr}$ postlesion and reaches a maximum (24-fold above normal) $18-24 \mathrm{hr}$ postlesion (White et al., 1987; White and Gall, 1986, 1987). During the period from 24 to $30 \mathrm{hr}$ after the lesion, enkephalin synthesis in the granule cell/mossy fiber system is elevated 14-fold above normal as measured by incorporation of ${ }^{35} \mathrm{~S}$ methionine into chromatographically identified methionine enkephalin (White et al., 1987). These increases are transient: preproenkephalin A mRNA levels in the dentate gyrus decline to near normal by $48 \mathrm{hr}$ postlesion. Thus, in the hilus lesion paradigm, increased enkephalin synthesis is initiated very early in the period of seizure activity and continues beyond the period of full paroxysmal electrographic discharges in hippocampus by at least $12 \mathrm{hr}$.

We have recently obtained data suggesting possible mechanisms through which these changes in enkephalin synthesis are triggered. Specifically, hilus lesions cause a large bilateral increase in ornithine decarboxylase (ODC) message and a 50-fold increase in ODC activity in hippocampus, and this seizuredependent induction begins within $3 \mathrm{hr}$ of the lesion (Baudry et al., 1986; C. Gall and J. White, unpublished observations). Increased ODC activity occurs in a number of tissues exposed to growth inducing treatments (Lewis et al., 1978), and the polyamines produced by ODC are known to accelerate the transcription of several varieties of RNA (Heby, 1981; Slotkin and Bartolome, 1986). Thus, it is not unlikely that the postlesion increases in preproenkephalin A mRNA are either the result of, or facilitated by, the earlier induction of ODC. Another potential intermediate to the regulation of enkephalin synthesis is suggested by our recent observation that mRNA for the protooncogene $c$-fos, suggested to be involved in surface signal transduction and growth processes in a number of systems (Milbrandt, 1986; Morgan and Curran, 1986), is also dramatically elevated by hilus lesion-induced seizures in adult rat hippocampus (White and Gall, 1987). Studies are currently in progress to determine the anatomical localization of the seizure-induced increases in ODC and $c$-fos message in hippocampus, and whether these inductions are necessary for the postseizure elevation in enkephalin synthesis in the dentate gyrus granule cells.

The processes responsible for the seizure-induced changes in mossy fiber dynorphin and CCK content are, as yet, unknown. It is possible that the mechanisms regulating peptide levels in the mossy fibers are linked in some way such that increased enkephalin synthesis or content suppresses the production of CCK or accelerates its catabolism. Such a negative cooperativity between enkephalin and CCK synthesis would be consistent with an observation made in a very different neuronal system. These peptides have been reported to undergo opposite seasonal variation in neuroendocrine cells of the cerebral ganglion of the freshwater snail: In the fall, these ncurons cxpress CCK, while in the spring they express enkephalin (Petrics-Gesser and Larsson, 1985). However, the results obtained here for mice treated with high doses of kainic acid indicate that in the mossy fiber system it is possible to dissociate the magnitude of the elevation of ENK-I from the depletion of CCK-I. Moreover, while we have found opposite variations in mossy fiber enkephalin and dynorphin immunoreactivities, intrastriatal kainic acid reportcdly induces a concurrent elevation of both enkephalin and dynorphin immunoreactivities in these axons. If linkages exist between the pathways regulating the different peptides, their expression or release may depend upon the type of physiological activity experienced by a particular class of cells.

The issue of the physiological consequences of shifts in the balance of peptides takes on added significance with the present observation that CCK-I and ENK-I are strongly and inversely affected by seizure-inducing conditions. Enkephalin and CCK have been demonstrated to exert antagonistic physiological effects in a number of systems (Zetler, 1979; Faris et al., 1983) and quite possibly have opposing influences over seizure activity. Enkephalin (injected i.c.v.) has epileptogenic properties (Frenk et al., 1978; Elazar et al., 1979), whereas CCK has been demonstrated to act as an anticonvulsant in several paradigms (Zetler, 1981; Kádár et al., 1984). This suggests that a shift in the balance of endogenous enkephalin and CCK within the hippocampal formation, most particularly a shift involving increased enkephalin and a complete loss of the anticonvulsant CCK from the mossy fibers, may underlie changes in seizure susceptibility.

The possibility that intense, but not seizure-producing, episodes of physiological activity elicit changes in mossy fiber peptides of the types reported here also deserves consideration. A substantial proportion of the granule cells fire at high rates for several seconds in rats engaged in locomotor activities during learning situations (Deadwyler at al., 1979; Rose et al., 1983), and periods of such activity are repeated across many training trials. It will be of interest to determine if electrical stimulation patterned after these natural granule cell activities elicits transient changes in neuropeptide balance in the mossy fiber system.

\section{References}

Baker, H., T. Kawano, F. L. Margolis, and T. H. Joh (1983) Transneuronal regulation of tyrosine hydroxylase expression in olfactory bulb of mouse and rat. J. Neurosci. 3: 69-78.

Baudry, M., G. Lynch, and C. Gall (1986) Induction of ornithine decarboxylase as a possible mediator of seizure-induced changes in genomic expression in rat hippocampus. J. Neurosci. 6: 3430-3435.

Blackstad, T. W., and A. Kjaerheim (1961) Special axo-dendritic synapses in the hippocampal cortex: Electron and light microscopic studies on the layer of mossy fibers. J. Comp. Neurol. 117: 133-159.

Blackstad, T. W., K. Brink, J. Hem, and B. Juene (1970) Distribution of hippocampal mossy fibers in the rat. An experimental study with silver impregnation methods. J. Comp. Neurol. 138: 443-450.

Charnay, Y., L. Léger, F. Dray, A. Bérnod, M. Jouvet, J. F. Pujol, and P. M. Dubois (1982) Evidence for the presence of enkephalin in catecholaminergic neurones of cat locus coeruleus. Neurosci. Lett. 30: 147-151.

Deadwyler, S. A., M. West, and G. Lynch (1979) Activity of dentate granule cells during learning: Differentiation of perforant path input. Brain Res. 169: 29-43.

Elazar, Z., E. Motles, Y. Ely, and R. Simantov (1979) Acute tolerance to the excitatory effect of enkephalin microinjections into hippocampus. Life Sci. 24: 541-548.

Faris, P., B. Komisuruk, L. Watkins, and D. Mayer (1983) Evidence for the neuropeptide cholecystokinin as an antagonist of opiate analgesia. Science 219: 310-312.

Fredens, K., K. Stengaard-Pedersen, and L.-I. Larsson (1984) Localization of enkephalin and cholccystokinin immunoreactivities in the 
perforant path terminal fields of the rat hippocampal formation. Brain Res. 304: 255-263.

Frenk, H., G. Urca, and J. C. Liebeskind (1978) Epileptic properties of leucine- and methionine-enkephalin: Comparison with morphine and reversibility by naloxone. Brain Res. 147: 327-337.

Fricke, R., and W. M. Cowan (1978) An autoradiographic study of the commissural and ipsilateral hippocampo-dentate projections in the adult rat. J. Comp. Neurol. 181: 253-270.

Gall, C. (1984a) The distribution of cholecystokinin-like immunoreactivity in the hippocampal formation of the guinea pig: Localization in the mossy fibers. Brain Res. 306: 73-83.

Gall, C. (1984b) Ontogeny of dynorphin-like immunoreactivity in the hippocampal formation of the rat. Brain Res. 307: 327-331.

Gall, C. (1984c) Seizure-producing treatments result in increased enkephalin and decreased cholecystokinin immunoreactivity in mossy fibers. Soc. Neurosci. Abstr. 10: 1112.

Gall, C. (1988) Localization and seizure-induced alterations of opioid peptides and CCK in the hippocampus. In Opioids in Hippocampus, J. F. McGinty, ed., N.I.D.A. Res. Monogr. (in press).

Gall, C., N. Brecha, H. J. Karten, and K.-J. Chang (1981a) Localization of enkephalin-like immunoreactivity to identified axonal and neuronal populations of the rat hippocampus. J. Comp. Neurol. 198: $335-350$.

Gall, C., N. Brecha, K.-J. Chang, and H. J. Karten (1981b) Localization of increased hippocampal leucine enkephalin-like immunoreactivity following hilar lesions in the adult rat. Soc. Neurosci. Abstr. 7: 94.

Gall, C., L. Berry, and L. Hodgson (1986) Cholecystokinin in the mouse hippocampus: Localization in the mossy fiber and dentate commissural systems. Exp. Brain Res. 62: 431-437.

Gall, C., J. Lauterborn, D. Burks, and K. Seroogy (1987) Colocalization of enkephalin and cholecystokinin in discrete areas of rat brain. Brain Res. 403: 403-408.

Gall, C., R. Pico, and J. Lauterborn (1988) Seizures induce distinct long-lasting changes in mossy fiber peptide immunoreactivity. Peptides 9: 79-84.

Greenwood, R. S., S. Godar, T. A. Reaves, Jr., and J. Hayward (1981) Cholecystokinin in hippocampal pathways. J. Comp. Neurol. 203: 335-350.

Heby, O. (1981) Role of polyamines in the control of cellular proliferation and differentiation. Differentiation 19: 1-20.

Hendry, S. H. C., and E. G. Jones (1986) Reduction in number of immunostained GABA neurons in deprived-eye dominance columns of monkey area 17. Nature 320:750-753.

Hong, J. S., P. L. Wood, J. C. Gillin, H. Y. T. Yang, and E. Costa (1980) Changes of hippocampal Met-enkephalin content after recurrent motor seizures. Nature 285: 231-232.

Iadarola, M. J., C. Shin, J. O. McNamara, and H.-Y. T. Yang (1986) Changes in dynorphin, enkephalin and cholecystokinin content of hippocampus and substantia nigra after amygdala kindling. Brain Res. 365: 181-191.

Kádár, T., A. Pesti, B. Penke, and G. Telegdy (1984) Inhibition of seizures induced by picrotoxin and electroshock by cholecystokinin octapeptides and their fragments in rats after intracerebroventricular administration. Neuropharmacology 23: 955-961.

Kanamatsu, T., J. F. McGinty, C. L. Mitchell, and J. S. Hong (1986a) Dynorphin- and enkephalin-like immunoreactivity is altered in limbic-basal ganglia regions of rat brain after repeated electroconvulsive shock. J. Neurosci. 6: 644-649.

Kanamatsu, T., J. Obie, L. Grimes, J. F. McGinty, K. Yoshikawa, S. Sabol, and J. S. Hong (1986b) Kainic acid alters the metabolism of met $^{5}$-enkephalin and the level of dynorphin $\mathrm{A}$ in the rat hippocampus. J. Neurosci. 6: 3094-3102.

Kanamatsu, T., C. D. Unsworth, E. J. Diliberto, Jr., O. H. Viveros, and J. S. Hong (1986c) Reflex splanchnic nerve stimulation increases levels of proenkephalin A mRNA and proenkephalin A-related peptides in the rat adrenal medulla. Proc. Natl. Acad. Sci. USA 83: $9245-$ 9249.

Kawano, T., and F. L. Margolis (1982) Transsynaptic regulation of olfactory bulb catecholamines in mice and rats. J. Neurochem. 39: 342-348.

Kilpatrick, D. L., R. D. Howells, G. Fliminger, and S. Udenfriend (1984) Denervation of rat adrenal glands markedly increases preproenkephalin mRNA. Proc. Natl. Acad. Sci. USA 81: 7221-7223.

LaGamma, E. F., J. E. Adler, and I. B. Black (1984) Impulse activity differentially regulates [leu] enkephalin and catecholamine characters in the adrenal medulla. Science 224: 1102-1 104.
LaGamma, E. F., J. D. White, J. E. Adler, J. E. Krause, J. F. McKelvy, and I. B. Black (1986) Depolarization regulates adrenal preproenkephalin mRNA. Proc. Natl. Acad. Sci. USA 82: 8252-8255.

Lason, W., B. Prezewlocka, L. Stala, and R. Prezewlocki (1983) Changes in hippocampal immunoreactive dynorphin and neoendorphin content following intra-amygdalar kainic acid induced seizures. Neuropeptides 3: 399-404.

Laurberg, S. (1979) Commissural and intrinsic connections of the rat hippocampus. J. Comp. Neurol. 184: 685-708.

Lewis, M. E., J. Laksmanian, J. Nagaiah, P. C. McDonnell, and G. Guroff (1978) Nerve growth factor increases activity of ornithine decarboxylase in rat brain. Proc. Natl. Acad. Sci. USA 75: 10211023.

McGinty, J. F., S. J. Henriksen, A. Goldstein, L. Terenius, and F. E. Bloom (1983) Dynorphin is contained within hippocampal mossy fibers: Immunochemical alterations after kainic acid administration and colchicine-induced neurotoxicity. Proc. Natl. Acad. Sci. USA 80. 589-593.

McGinty, J. F., T. Kanamatsu, J. Obie, R. S. Dyer, C. L. Mitchell, and J. S. Hong (1986) Amygdaloid kindling increases enkephalin-like immunoreactivity but decreases dynorphin-A-like immunoreactivity in rat hippocampus. Neurosci. Lett. 71: 31-36.

Meyer, D. K., R. Widmann, and G. Sperk (1986) Increased brain levels of cholecystokinin octapeptide after kainic acid-induced seizures in the rat. Neurosci. Lett. 69: 208-211.

Milbrandt, J. (1986) Nerve growth factor rapidly induces c-fos mRNA in PC12 rat pheochromocytoma cells. Proc. Natl. Acad. Sci. USA 83. 4789-4793.

Morgan, J. I., and T. Curran (1986) Role of ion flux in the control of c-fos expression. Nature 322: 552-555.

Nadi, N. S., R. Head, M. Grillo, J. Hempstead, J. Grannot-Reisfeld, and F. Margolis (1981) Chemical deafferentation of the olfactory bulb: Dopamine and norepinephrine. Brain Res. 213: 365-377.

Petrics-Gesser, B., and L.-I. Larsson (1985) Changes from enkephalinlike to gastrin/cholecystokinin-like immunoreactivity in snail neurons. J. Neurosci. 5: 1412-1417.

Rose, G., D. Diamond, and G. S. Lynch (1983) Dentate granule cells in the rat hippocampal formation have the behavioral characteristics of theta neurons. Brain Res. 266: 29-37.

Slotkin, T. A., and J. Bartolome (1986) Role of ornithine decarboxylase and the polyamines in nervous system development: A review. Brain Res. Bull. 17: 307-320.

Sloviter, R. S., and B. P. Damiano (1981) On the relationship between kainic acid-induced epileptiform activity and hippocampal neuronal damage. Neuropharmacology 20: 1003-1011.

Sperk, G., R. Wieser, R. Widmann, and E. A. Singer (1986) Kainic acid induced seizures: Changes in somatostatin, substance $P$ and neurotensin. Neuroscience 17: 1117-1126.

Stengaard-Pedersen, K., K. Fredens, and L.-I. Larsson (1983) Comparative localization of enkephalin and cholecystokinin immunoreactivities and heavy metals in the hippocampus. Brain Res. 273: 81-96.

Sternberger, L. (1979) Immunohistochemistry, 2nd ed., Wiley, New York.

Unger, A., and J. H. Phillips (1983) Regulation of the adrenal medulla. Physiol. Rev. 63: 787-843.

Vindrola, R. Briones, M. Asai, and A. Fernandez Guardiola (1981) Amygdaloid kindling enhances the enkephalin content in rat brain. Neurosci. Lett. 21: 39-44.

Vindrola, O., M. Asai, M. Zubieta, E. Talaviera, E. Rodriquez, and G. Linares (1984) Pentylenetetrazol kindling produces a long-lasting elevation of IR-met-enkephalin but not IR-leu-enkephalin in rat brain. Brain Res. 297: 121-125.

White, J. D., and C. M. Gall (1986) Increased enkephalin gene expression in hippocampus following seizures. In Progress in Opioid Research (NIDA Res. Monogr. 75), J. W. Holaday, P.-Y. Law, and A. Herz, eds., pp. 393-396.

White, J. D., and C. M. Gall (1987) Differential regulation of neuropeptide and proto-oncogene mRNA content in the hippocampus following recurrent seizures. Mol. Brain Res. 3: 21-29.

White, J. D., C. M. Gall, and J. F. McKelvy (1987) Enkephalin biosynthesis and enkephalin gene expression are increased in hippocampal mossy fibers following a unilateral lesion of the hilus. J. Neurosci. 7: 753-759.

Wilson, S. P., K.-J. Chang, and O. H. Viveros (1982) Proportional secretion of opioid peptides and catecholamines from adrenal chromaffin cells in culture. J. Neurosci. 8: 1150-1156. 
Yoshikawa, K., J.-S. Hong, and S. L. Sabol (1985) Electroconvulsive shock increases preproenkephalin messenger RNA abundance in rat hypothalamus. Proc. Natl. Acad. Sci. USA 82: 589-593.

Zahm, D. S., L. Zaborszky, V. E. Alones, and L. Heimer (1985) Evidence for the coexistence of glutamate decarboxylase and Met-enkephalin immunoreactivities in axon terminals of rat ventral pallidum. Brain Res. 325: 317-321.
Zetler, G. (1979) Antagonism of cholecystokin-like peptides by opioid peptides, morphine or tetrodotoxin. Eur. J. Pharmacol. 60: 67-77.

Zetler, G. (1981) Anticonvulsant effects of caerulein, cholecystokinin octapeplide (CCK-8) and diazepam against seizures produced in mice by harman, thiosemicarbazide and isoniazid. Neurosci. Lett. $24: 175$ 182. 